\title{
A Multi-Burst Sliding Encoding for Mobile Satellite TV Broadcasting
}

\author{
Mohamed Amine Ismail \\ UDcast \\ Sophia Antipolis, France \\ Email: amine.ismail@udcast.com
}

\author{
Walid Dabbous \\ INRIA \\ Sophia Antipolis, France \\ Email: walid.dabbous@inria.fr
}

\author{
Antoine Clerget \\ UDcast \\ Sophia Antipolis, France \\ Email: antoine.clerget@udcast.com
}

\begin{abstract}
Protection of data against long fading time is one of the greatest challenges posed by a satellite delivery system offering multimedia services to mobile devices like DVB-SH. To deal with this challenge several enhancements and modifications of the existing terrestrial mobile TV (DVB-H) are being considered. These solutions provide the required protection depth but they don't take into account the specificity of mobile handheld devices such as power consumption, memory constraints and chipsets implementation costs. In this paper, we propose an innovative algorithm (called Multi Burst Sliding Encoding or MBSE) that extends the DVB-H intra-burst (MPE-FEC) protection to an inter-burst protection so that complete burst losses could be recovered while taking into account the specificity of mobile handheld devices. Based on a clever organisation of the data, our algorithm allows to provide protection against long term fading while still using RS code implemented in DVB-H chipsets. We evaluate the performance of MBSE by both theoretical analysis as well as intensive simulations and experiments. The results also show good performance in terms of protection, battery and memory saving. The MBSE is now under standardisation and it is considered by the DVB Forum as the main solution for the DVB-SH class terminals.
\end{abstract}

\section{INTRODUCTION}

Reliable video streaming over unreliable, bandwidthconstraint, unidirectional broadcast links for mobile users is a challenging task. Reliability in this environment is achieved using Forward Error Correction (FEC).

DVB-H [1] the Digital Video Brodacsting system for Handheld, is based on the digital terrestrial television standard DVB-T [2] and takes into account the specific properties of typical handheld size terminals: limited battery capacity and mobile reception using small antenna with a low gain. To solve the problem of the power consumption, a time division multiplex transmission is introduced at the link layer level. This technique is called Time-Slicing. The key idea is to transmit data in "bursts" exclusively carrying a single service. This enables a time selective reception of the wanted service by switching to a power-save mode during the transmission of other services. On the other hand the coding operations of DVB-T (Reed-Solomon code and convolutional code) already providing a sifficient protection in stationary channels becomes not suitable to offer a good transmission quality for mobile users in Typical Urban channel profile (TU-6) ${ }^{1}$. For this reason a link layer FEC called MPE-FEC (Multi Protocol Encapsulation- Forward Error Correction) [3] was introduced. The MPE-FEC, called also Inner-FEC, is the second main innovation of DVB-H besides the time slicing. It consists in a Reed-Solomon (RS) code applied to a specific frame structure called the MPE-FEC frame. The MPE-FEC error protection is calculated separately for each individual service.

DVB-SH [4] is a new standard defining the way to deliver "IP based media content and data" to handheld terminals using hybrid satellite/terrestrial solution. It takes into account the specific properties of a direct satellite link for a mobile reception which introduces a new channel modeled often by an "LMS" (Land Mobile Satellite) model ${ }^{2}$. This kind of channel is characterized by long fading time where the signal can be interrupted for several seconds. The DVB-H MPE-FEC mechanism corrects errors within a burst with a maximum size of 1.5 Mbits ( 1 second) and is not adapted for networks with long loss duration ( $\sim$ tens of seconds).

In this paper, we propose a new algorithm called "Multi Burst Sliding Encoding " to offer a good transmission quality in satellite broadcasting systems for mobile devices, taking into account the power consumption and the memory constraint. This algorithm is capable of generating an outer FEC able to recover several consecutive lost bursts. It is compatible with the existing DVB-H standard and FEC computation can use the same RS $(191,255)$ algorithm and can thus benefit from the existing hardware. The proposed encoding and decoding algorithms were implemented and several experiments were performed to evaluate the performance of the proposed solution.

The rest of the paper is organized as follows: section II describes the possible solutions to avoid long fading time and explains why they are not suitable for DVB-SH handheld class receivers. Then the "Multi Burst Sliding Encoding" is presented in section III. In section IV, we show the experimental performance of our algorithm applied to the RS $(191,255)$

\footnotetext{
${ }^{1}$ TU-6 models the terrestrial propagation in an urban area. It uses 6 resolvable paths, each one characterized by a delay, a power and a fading model.

${ }^{2}$ In satellite to mobile communications, The spectral and statistical properties of the signal are influenced by shadowing caused by obstacles such as trees, houses or small buildings.
} 
for TU6 and LMS channels. Finally section V presents our conclusions and remarks.

\section{Possible SOlutions to AVOID LONG FADING TIME}

In order to provide a good quality streaming service over a channel with long fading it is necessary to operate the protection over a large block of data and to spread protected data and FEC over the time. A such long time encoder and interleaver can be located at the physical or at the data link layer. The two solutions are presented.

\section{A. Physical layer long interleaver}

Time interleaving consists in dispersion of data over time: protected data are sent in several discrete bursts to disperse losses. With physical layer interleaving information received during poor reception condition are combined with information received during good reception conditions. If enough FEC is available, the original data can be recovered although parts have been erased by the channel. The adequate interleaver length depends on the type of channel considered. For a satellite channel, slow fading is the dominant factor and the interleaver lengths to be considered are in the order of several seconds [5], [6]. Reference [7] proposes an architecture of a long time interleaver for DVB-SH class receivers. In the receiver side the decoding process can be achieved by using either a high-complexity soft decision decoding or a lower performance, lower complexity hard decision decoding [8]. As it provides higher recovering capacity of the physical layer interleaver, only the soft decision decoding (SDD) will be considered. In SDD, the decoder front-end does not make a hard decision about whether a 0 or 1 bit was transmitted but rather makes a soft decision corresponding to the distance between the received symbol and the symbol corresponding to a 0-bit or a 1-bit transmission. The demodulator produces an integer (coded on $\mathrm{N}$ bits) for each bit in the data stream. This integer could be seen as a measure of how likely it is that the bit is a 0 or a 1 and is also called soft bits. Thus the soft bits should be stored in the de-interleaver in order to be used to make better decisions about the transmitted codeword.

However, the price to achieve the benefits of a long interleaver with a soft-decision is a higher memory usage on the receiver side. The memory consumption of a time interleaver is the product of the interleaver length (seconds) multiplied by the bit rate (bits per second) multiplied by the number of the bit-decision divided by the number of bits transmitted per symbol (1 for BPSK, 2 for QPSK, 4 for 16QAM, 6 for 64QAM...). For example at a bitrate of $12 \mathrm{Mbps}$, an interleaver length of 20s, using a decoder with 12-bit decision and QPSK modulation, the required on-chip memory for the de-interleaver is 1440 Mbits. This type of memory is quite expensive to implement.

Another considerable drawback of this solution is the loss of the power saving which derives from the time slicing since several services are interleaved together at the bit level and the decoder has to receive all services and extract the desired one after the deinterleaving operation.
For these reasons, even if the physical layer long interleaver can provide the required quality for the transmission, it is not suitable for handheld receivers with memory and battery limitations.

\section{B. Link layer block encoding}

Another way to enhance quality over channels with long fading is to apply a link layer protection mechanism. Such mechanism is mainly based on an extension of the encoding matrix size from one burst (in the DVB-H case) to several bursts and is using a large block FEC encoding mechanism for providing the protection [9].

The FEC encoder allocates an encoding matrix with a size of $b$ bursts made of $C$ data columns each. For each $b$ bursts, FEC columns are computed and then sent within the next $b$ bursts ( $F_{o}$ FEC columns are sent with each burst).

With this mechanism if a whole block is lost then the FEC columns of the previous block are lost too. So to compute the recovery capacity of this mechanism we have to compute the minimum number of bursts belonging to the same block that must be received to enable the decoder recovering all bursts from the previous block. To recover the previous block the decoder needs to receive b.C FEC columns which represent $\frac{b . C}{F_{o}}$ bursts. This makes sense only if $\frac{C}{F_{o}} \leq 1$. Therefore the decoder can recover up to $n$ successive bursts such that $(2 b-n) \frac{F_{o}}{C} \geq b$. This implies that $n \leq\left\lfloor 2 b .\left(1-\frac{C}{2 F_{0}}\right)\right\rfloor$. This expression is bounded by $(M-1)$ where $M=2 b$ is the decoder memory usage.

Even if this mechanism can provide the required protection depth it suffers from its "block" nature, and becomes suboptimal for our target application for the reasons below:

1) To decode a FEC block made of $k$ source symbols and $(n-k)$ parity symbols the decoder needs to receive any $k$ among the $n$ symbols. If the decoder receives $D=k-L$ source symbols and $F<L$ parity symbols, then no symbols can be recovered, i.e. if $\mathrm{R}$ defines the number of recovered symbols the probability $P((R>0) \mid(L>$ $F))=0$. Now we divide this FEC block into $N$ smaller blocks each one made of $\frac{k}{N}$ source symbols and $\frac{n-k}{N}$ parity symbols. If the decoder receives for each small block $d_{i}=\frac{k}{N}-l_{i}$ data symbols and $f_{i}$ parity symbols such that $\sum_{i=0}^{N-1} d_{i}=D, \sum_{i=0}^{N-1} f_{i}=F$ and $\sum_{i=0}^{N-1} l_{i}=L$. The probability to recover $R$ source symbols among the $L$ lost ones becomes:

$$
\begin{gathered}
P((R>0) \mid(L>F))= \\
\sum_{i=0}^{N-1}\left(\begin{array}{c}
N \\
i
\end{array}\right) P\left(R_{i}>0\right)^{i} P\left(R_{i}=0\right)^{N-i} \\
=1-P\left(R_{i}=0\right)^{N} \geq 0
\end{gathered}
$$

$P\left(R_{i}\right)$ is the probability to recover $R_{i}$ source symbols in a small block.

where $(L-F)<\frac{k \cdot(N-1)}{N}$ yields that (1) is strictly greater than 0 . In other words, large block FEC coding is not always better than small FEC blocks, and especially 
when the available FEC ratio $\left(\frac{F E C}{D A T A+F E C}\right)$ is less than the Packet Loss Rate (PLR) of the channel, or when the constraints on the width of the block (memory, delay,...) imply a lot of variability of the PLR over the blocks.

2) In the case of packets loss, the decoder needs to receive the full block before the decoding starts. This induces a fixed delay on the reception time.

3) The end-to-end delay is bounded by $2 \times$ protection_period + decoding_delay where the decoding delay depends on the chosen FEC code [10]. For Reed Solomon it increases quadratically according to block size, and even if for some other codes like Raptor it increases linearly, it remains quite important, as shown in reference [11].

4) In handheld devices, the FEC decoding algorithm is implemented in a specific hardware because of its processing complexity. The chipset implementing the FEC decoder needs a fast memory buffer whose size is a little larger than the size of the FEC block (data + meta-data). This type of memory is quite expensive to implement.

\section{The Multi Burst SLiding Encoding}

In order to provide a better solution for TV broadcasting under a channel with a long fading time for a DVB-SH handheld class receiver, we propose a new mechanism called "Multi Burst Sliding Encoding" MBSE. The sliding encoding mechanism is a link layer solution for extending the duration of the protection while still using small size blocks. It extends the existing DVB-H MPE-FEC intra-burst protection to an inter-burst protection so that complete burst losses may be recovered by producing an "outer FEC". In order to achieve this protection, data coming from several bursts are interleaved inside parallel matrices before FEC protection is applied, even if original data are not sent interleaved eventually. On the reception side, received data are also interleaved before being placed in decoding matrices. In order to achieve this extension we need the following parameters:

- $B$ : The encoding parallelization factor expressed in encoding matrix units. Each burst is split into $B$ parts distributed over $B$ encoding matrices.

- $S$ : The depth of the FEC spreading factor, It means that produced FEC is spread over $S$ bursts.

- $F_{o}$ : Number of computed outer-FEC columns from an encoding matrix.

The algorithm works as follows: The encoder allocates a set of $B+S$ encoding matrices divided into two classes : $B$-class made of $B$ matrices and $S$-class made of $S$ matrices. At each iteration a sliding window (hence the name of the algorithm) is applied to the $B+S$ matrices and thus one matrix from the $B$-class goes to the $S$-class and vice versa.

Each data burst is considered as a matrix of bytes made of $C$ data columns. Each matrix is divided into $B$ parts made of a number of non contiguous columns taken in a particular way. Each part is placed in a specific encoding matrix from the $B$ class. At each iteration only one specific encoding matrix form the $S$-class containing columns coming from several bursts

\begin{tabular}{|c|c|c|c|}
\hline Case & C/N & Environment & Speed \\
\hline \hline sub-50-6 & $6 \mathrm{db}$ & LMS & $50 \mathrm{Km} / \mathrm{h}$ \\
\hline su-50-10 & $10 \mathrm{db}$ & LMS & $50 \mathrm{Km} / \mathrm{h}$ \\
\hline sub-3-6 & $6 \mathrm{db}$ & LMS & $3 \mathrm{Km} / \mathrm{h}$ \\
\hline sub-3-10 & $10 \mathrm{db}$ & LMS & $3 \mathrm{Km} / \mathrm{h}$ \\
\hline Tu6-3-5 & $5 \mathrm{db}$ & Terrestrial urban & $3 \mathrm{Km} / \mathrm{h}$ \\
\hline Tu6-5-3.3 & $3.3 \mathrm{db}$ & Terrestrial urban & $5 \mathrm{Km} / \mathrm{h}$ \\
\hline Tu6-50-4 & $4 \mathrm{db}$ & Terrestrial urban & $50 \mathrm{Km} / \mathrm{h}$ \\
\hline \multicolumn{4}{|c}{ Table I } \\
\hline
\end{tabular}

RECEIVE SCENARIOS

is encoded and thus $F_{o}$ FEC columns are generated. Each burst is sent as is without modification and without additional delay. At the end of the burst some FEC columns, chosen in a specific way from the $S$-class matrices, are appended and sent. Receiver not supporting MBSE will just ignore the appended FEC bytes. [11].

The detailed algorithm of MBSE is presented in reference

In reference [11] we have analysed the recovery capacity of the multi-burst sliding encoding. In fact MBSE is able to recover up to $\frac{M}{\left(\frac{C}{F_{o}}+1\right)}$ consecutive lost bursts $(M=B+S)$. For large enough FEC percentage $\left(\frac{F_{o}}{C}\right)$ This expression ( bounded by M) can reach $(M-1)$. This means that MBSE has the same maximum recovering capacity as the large block encoding which is also $(M-1)$ consecutive lost bursts as shown in section II-B.

Since only one matrix among $\mathrm{M}$ has to be decoded at a time, the size of the required fast memory (on chip) is only one burst, which is also the case of the DVB-H terminals. The rest of the memory can be allocated at the host level.

\section{Experimental Performance of MBSE}

\section{A. Trace files based simulations}

In the following section we have applied both the RS $(191,255)$ MBSE and the ideal block encoding algorithms (optimistic results for block encoding) to some link layer DVB-SH trace files implementing different reception scenarios provided by the DVB-SSP work group[12]. The aim is to compare their performance recovery according to Errored Second Ratio 5\% (ESR5) ${ }^{3}$ and the Packet Loss Rate (PLR).

1) Simulation tools: A simulation code written in C Linux process the dump files and applies the two decoding algorithms. For the MBSE it takes as input the $\mathrm{B}+\mathrm{S}$ parameters and the FEC ratio, and for the block encoding algorithm the block size and the FEC ratio. It gives for each trace file the PLR and the ESR5. The dump files implement different receive scenarios. each scenario is characterized by a carrier to noise ratio $(\mathrm{C} / \mathrm{N})$, a propagation environment (LMS sub-urban or terrestrial urban) and a receiver speed. For the simulation the following assumption are done: IP packet size is set to 1000 bytes and the FEC ratio is fixed to $33 \%$. The considered receive scenarios are given by table $\mathrm{I}$.

\footnotetext{
${ }^{3}$ ESR: seconds with errors over an observation period. ESR5 corresponds to 1 second with error over a 20 seconds observation period.
} 


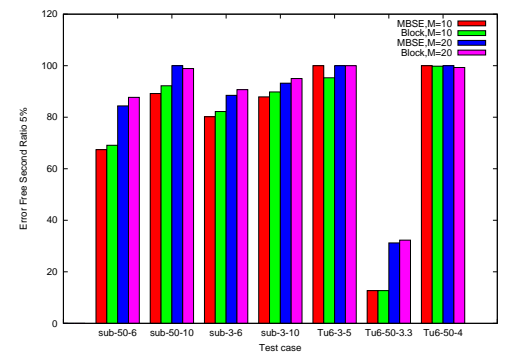

Figure 1. 1-ESR5 MBSE versus ideal Block

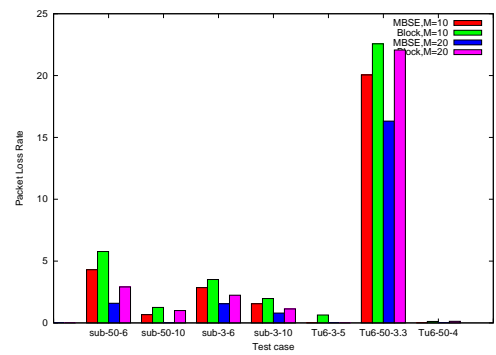

Figure 2. PLR MBSE versus Block

2) Simulation results: Figure 1 reports the 1-ESR5 (or EFSR5 Error Free Second Ratio 5\%) performance for both the MBSE with Reed Solomon $(191,255)$ and the large block encoding with an ideal code. When comparing the performance of the two encoding algorithm for the same memory usage (M) according to the EFSR5 criteria we can remark that there is not a significant difference between them and the two algorithms have a very closely near performance (the difference is under $2 \%$ ). Regarding Figure 2 which reports PLR we can remark that MBSE performs better than block encoding and this can be explained by the fact that the block encoding can not decode the block when the number of packet losses is greater than the available FEC packets while the MBSE can decode some small blocks.

\section{B. Experiments with an emulated link}

In the following we applied the RS(191,255) MBSE algorithm to a live stream while varying the $\mathrm{C} / \mathrm{N}$ and the $\mathrm{B}+\mathrm{S}$. The aim is to evaluate the gain provided by the MBSE compared to DVB-T and DVB-H.

1) Test bed description: The encoding and decoding algorithms of the MBSE are implemented in the UDcast DVB-SH IP-Encapsulator and in the UDcast TS-Processor respectively. MPE and MPE-OFEC sections are generated so that an IP datagram forms the payload of an MPE-section and an outerFEC column forms the payload of an MPE-OFEC section. The MPE and MPE-OFEC sections are then fragmented into MPEG2 packets carried through an ASI cable to a DVBSH COFDM modulator. The modulated signal was passed through a hardware channel emulator [13] implementing the TU6 multipath channel model and the Land Mobile Satellite LMS model. The Noisy signal is demodulated by the DVBSH signal demodulator and the generated MPEG2 packets are passed to the MBSE decoder to generate the IP datagrams. An

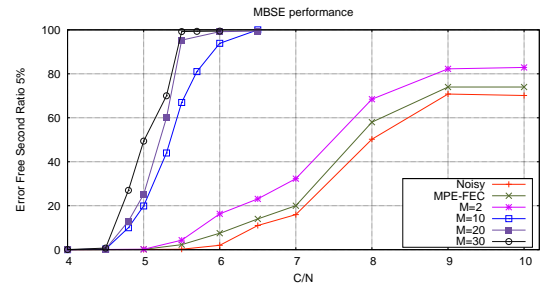

Figure 3. Error Free Second Ratio 5\% in an LMS channel for a FEC ratio of $33 \%$ while varying the memory usage

IP analyzer gives statistics on the PLR, the Errored Second Ratio (ESR) and the EFSR5. For the experiments the physical modulation is set to QPSK, the physical layer FEC ratio is set to $50 \%$, the physical layer interleaver lengt is set to $100 \mathrm{~ms}$ and the MBSE FEC ratio is set to $33 \%$.

2) Experiments Results: Figures 3, 4 and 5 show respectively the EFSR5, the PLR and the ESR for the MBSE algorithm with different value of $\mathrm{B}+\mathrm{S}$ while varying the carrier to noise ratio in an LMS channel model for a mobile receiver at $3 \mathrm{Km} / \mathrm{h}$. From these set of curves, several interesting observations can be drawn. Firstly we can remark that in an LMS channel the DVB-H MPE FEC is not usable since it can not provide a good transmission quality even if we use a high transmission power, the service availability (EFSR5) is bounded by $\sim 80 \%$. Secondly the higher $\mathrm{M}(\mathrm{B}+\mathrm{S})$ the better the performance of the MBSE scheme in terms of EFSR5, PLR and ESR; for example an EFSR of $99 \%$ can be reached either by using a $\mathrm{B}+\mathrm{S}=10$ at $6.5 \mathrm{db}$ or a $\mathrm{B}+\mathrm{S}=30$ at $5.3 \mathrm{db}$. Finally we can observe a better performance of the MBSE with $M=2$ (no data interleaving) comparing to MPE-FEC mechanism. This can be explained by the fact that in the MBSE the FEC of a given data burst is sent with the next burst while in MPE-FEC the FEC and the corresponding data are sent in the same burst. Regarding Figures 7, 8, 9 which report respectively the EFSR5, the PLR and the ESR while varying the $\mathrm{C} / \mathrm{N}$ in a TU6 channel model for a mobile receiver at $3 \mathrm{Km} / \mathrm{h}$, we can remark that even if MBSE was designed for an LMS channel, it can improve significantly the performance of the receiver in term of data recovering under a terrestrial transmission (TU6) and thus it can be interesting for DVB$\mathrm{H}$, WiMAX and other burst based transmission protocols. Considering a service availability ratio (1-ESR5) higher than 99\% we can remark that with MBSE using $\mathrm{M}=30$ we can achieve a gain of $\sim 3 \mathrm{db}$ compared to a receiver without a link layer FEC mechanism and a gain of $\sim 2 \mathrm{db}$ compared to a DVB$\mathrm{H}$ MPE-FEC mechanism. This gain in terms of $\mathrm{C} / \mathrm{N}$ can be used by the broadcasters to increase the coverage area of the transmitters or to reduce the transmission power of the base stations while maintaining the same coverage area and the same quality of service. It can also help to save the bandwidth as the required protection can be provided by increasing the $\mathrm{B}+\mathrm{S}$ factor (memory usage) while decreasing the FEC ratio as shown by the Figure 6 . 


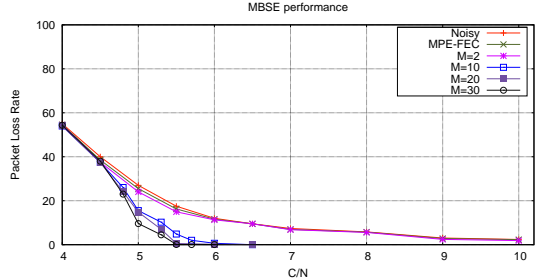

Figure 4. Packet Loss Rate in an LMS model for a FEC ratio of $33 \%$ while varying the memory usage

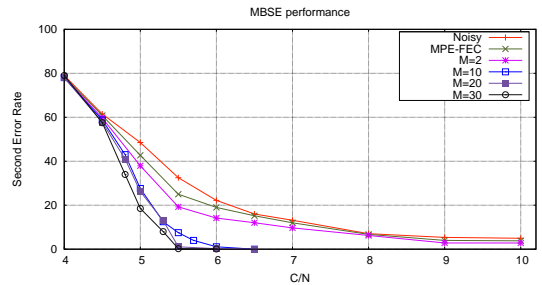

Figure 5. Errored Second Ratio in an LMS model for a FEC ratio of $33 \%$ while varying the memory usage

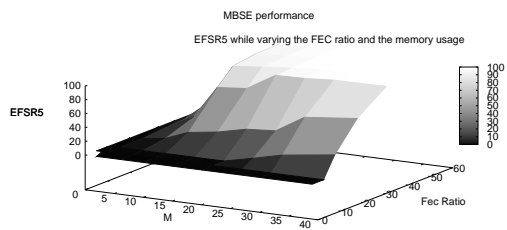

Figure 6. Error Free Second Ratio 5\% in an LMS channel at 5.5db while varying the FEC ratio and the memory usage

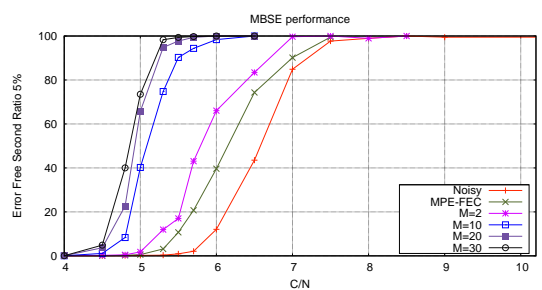

Figure 7. Error Free Second Ratio 5\% in a TU6 channel for a FEC ratio of $33 \%$ while varying the memory usage

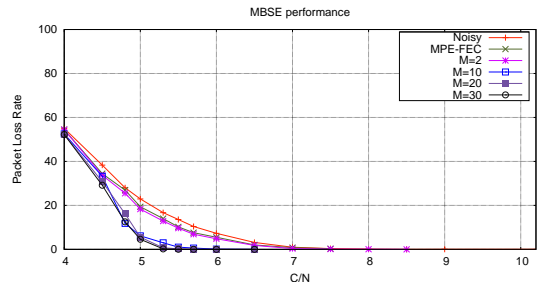

Figure 8. Packet Loss Rate in a TU6 channel for a FEC ratio of $33 \%$ while varying the memory usage

\section{CONCLUSION}

In this paper we have proposed an innovative algorithm called MBSE (Multi Burst Sliding Encoding) performing a sliding encoding at the link layer level to protect transmitted data against long fading time. We have derive an analytic expression to study the recovery capacity of our solution then we

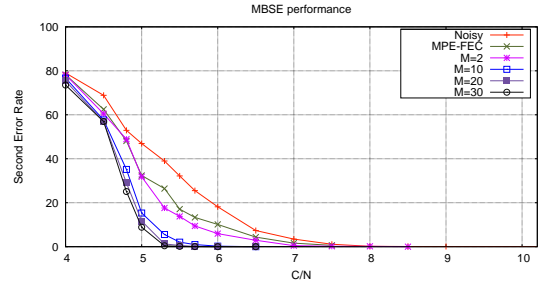

Figure 9. Errored Second Ratio in a TU6 channel for a FEC ratio of 33\% while varying the memory usage

validated it by simulations and by a real implementation and experiments. It offers significant advantages comparing to the other solutions. Our algorithm was approved by the TM-SSP DVB work group, it is considered as the main solution for the DVB-SH class terminals and it is now under standardization by the DVB-Forum as MPE-iFEC (i for inter-burst) [14]. Several implementations of the MBSE are currently in progress and some tests on field will start in the next months. As a future work, we intend to study a recursive decoding between the MPE-FEC and MBSE for DVB-SH, and we will adapt our current disign for a better protection of variable bitrate flows. Finally, we will consider the interest of MBSE for the WiMAX Multicast and Broadcast Service (MBS) and for some other burst-based technologies.

\section{REFERENCES}

[1] Transmission System for Handheld Terminals (DVB-H), Digital Video Broadcasting (DVB) Std. ETSI (EN) 302 304, Nov. 2004, release 1.1.1.

[2] Framing Structure, Channel Coding and Modulation for Terrestrial Television, Digital Video Broadcasting (DVB) Std. ETSI (EN) 300 744, Nov. 2004, release 1.5.1.

[3] DVB-H Implementation Guide-lines, Digital Video Broadcasting (DVB) Std. ETSI TR 102 377, Nov. 2005, release 1.2.1.

[4] System Specifications for Satellite Services to Handheld Devices (SH) Below $3 \mathrm{GHz}$, Digital Video Broadcasting (DVB) Working Draft Proposed Standard DVB-SSP0162, Rev. 5, Jan. 2007, release 1.6.2.

[5] M. Akhter, "Performance of Channel Interleaved Turbo Coded System for High Rate Mobile Satellite Communications," in Personal, Indoor and Mobile Radio Communications (PIMRC 2000), London, Untied Kingdom, Sep. 2000.

[6] S. Cioni, G. E. Corozza, M. Neri, and A. Vanelli-Coralli, "On the Use of OFDM Radio Interface for Satellite Digital Multimedia Broadcasting Systems," International Journal Of Satellite Communication And Networking, vol. 24, no. 2, 2006.

[7] C. Keip, "Physical Layer Interleaving and FEC," Digital Video Broadcasting (DVB), Tech. Rep. DVB-SSP0019, May 2006.

[8] K. Lee, D. S. Hani, and K. Kim, "Performance of the Viterbi Decoder for DVB-T in Rayleigh Fading Channel," IEEE Trans. Consum. Electron., vol. 44, no. 3, Aug. 1998.

[9] T. Stockhammer, "Upper Layer FEC for DVB-SH with Raptor Code," Digital Video Broadcasting (DVB), Tech. Rep. DVB-SSP0251, Jun. 2007.

[10] O. A. U. Demir, "Raptor Versus Reed Solomon Forward Error Correction Codes," International Symposium on Computer Networks, pp. 264-269, Jun. 2006.

[11] M. Ismail, W. Dabbous, and A. Clerget, "A Multi-Burst Sliding Encoding for Mobile Satellite TV Broadcasting," Institut National de Recherche en Informatique et en Automatique (INRIA), Tech. Rep. (RR6785), Dec. 2008.

[12] DVB Forum home page. [Online]. Available: http://www.dvb.org

[13] O. Rousset and J. L. Pavy, SSH-2000_DVB-SH Signal Generator Technical Specification, Teamcast, Mar. 2008, release 4.3.

[14] DVB-SH Implementation Guidelines, Digital Video Broadcasting (DVB) Working Draft Proposed Standard DVB-SSP 0252r9f, Rev. 14, Apr. 2008. 